\title{
Bandwidth Allocation DBA (BA-DBA) Algorithm for XPON Networks
}

\author{
Karamitsos loannis \\ Telecom Lab,On Telecoms \\ Athens, Attica \\ 16342, Greece
}

\author{
Al-Arfaj Khalid \\ Rayadah Investment Co \\ Riyadh, KSA \\ Kingdom of Saudi Arabia
}

\begin{abstract}
Passive Optical Networks (PONs) technology is considered as a promising solution for the next-generation broadband access network. In this paper we present different dynamic bandwidth algorithms (DBA) and estimate the bandwidth between OLT and ONT in order to improve network efficiency and packet processing. We show that queuing delays under dynamic bandwidth allocation algorithm results in an unexpected behavior for certain traffic classes and with the our proposed algorithm (BA-DBA) alleviate this inappropriate behavior. We conduct detailed simulations experiments to study the performance and the throughput of the proposed algorithms. Through respective simulations, it is estimated that the average queue size, the packet delay and the throughput of the proposed BA-DBA algorithm is performed better than a common SR-DBA algorithm.
\end{abstract}

\section{General Terms}

Optical Networks, Passive Optical Networks, Bandwidth

\section{Keywords}

Bandwidth, DBA, Optical Networks, PON.

\section{INTRODUCTION}

Passive Optical Network (PON) [1] is a point-to-multipoint optical network and all transmissions in the PON are performed between an Optical Line Terminal (OLT) and Optical Network Units (ONUs). In the upstream direction, the ONUs need to share the channel capacity by allocating the bandwidth to each ONU as described in the following figure.1. One of the main challenges of a Passive Optical Networks (PON) is to schedule the transmissions and the bandwidth allocation in the upstream channel efficiently.

In the past, many researchers studied and developed appropriate scheduling algorithms in terms of efficiency, support QoS of each traffic flow and optimistic bandwidth allocation to the users reducing delay and jitter. There are two main techniques used in the PON networks such as the fixed bandwidth allocation (FBA) and the dynamic bandwidth allocation (DBA) [2].

The first technique allocates the same transmission slots to every Optical Network Unit (ONU) in every service cycle. It is simple scheme, but, it does not perform optimally.

The second technique allocates dynamically the transmission in the upstream channel based on each ONU's requested bandwidth; therefore the dynamic scheme provides a more realistic, efficient, and flexible bandwidth allocation.

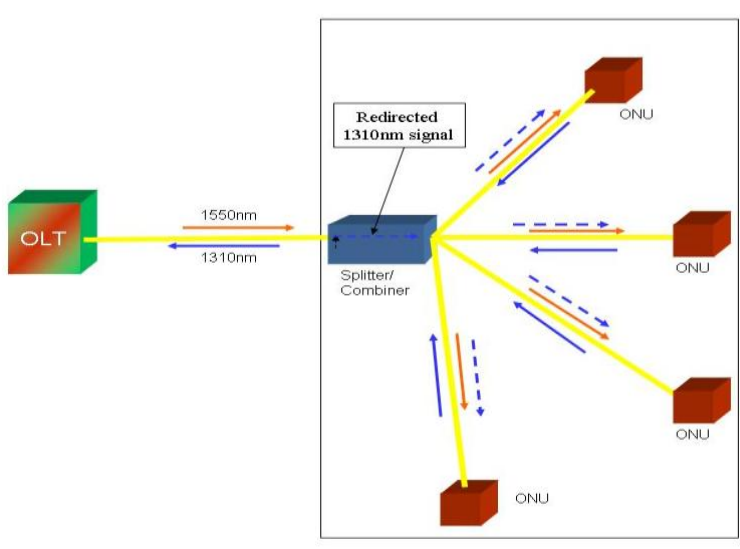

Fig. 1: PON typical configuration

This paper is organized as follows: In Section 2 a description of DBA scheduling algorithms are discussed. The common used SR-DBA algorithm in Section 3 is presented. In Section 4, the proposed BA_DBA algorithm is being illustrated. Finally, the performance simulations results from the comparisons between the SR-DBA and BA-DBA proposed algorithms are presented in the Sections 5 and 6.

\section{DBA SCHEDULING ALGORITHMS}

In this section, DBA scheduling algorithms proposed for PON networks can be categorized in different ways. DBA algorithms may be either centralized or distributed scheduling.

In the centralized scheduling the Optical Line Terminal (OLT) computes the bandwidth allocation centrally, which is the most common approach. The Interleaved Polling with Adaptive Cycle Time (IPACT) [3] is a very common centralized algorithm.

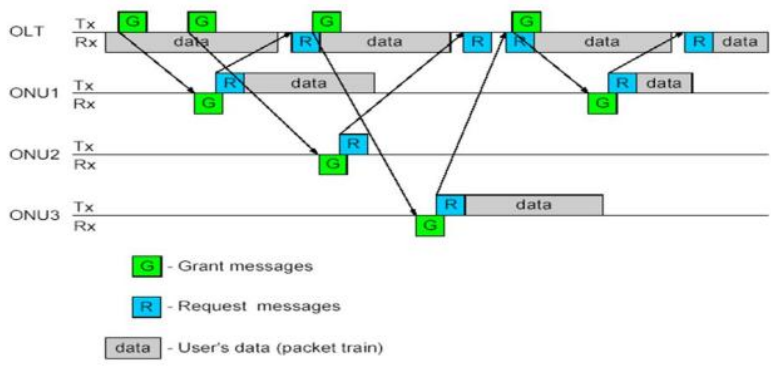

Fig. 2: Interleaved Polling with Adaptive Cycle [3] In the IPACT mechanism the cycle period adjusts to the bandwidth requirements of the ONUs and the definition of 
maximum transmission window does not allow ONUs with high traffic level to monopolize the bandwidth resource. The most important in this mechanism is that IPACT uses an interleaving polling approach [5], in which the next ONU is polled before the transmission of the previous one is finished in order to utilize the efficiency of the channel.

The IPACT grant sizing is performed using five different alternatives: fixed, limited, gated, constant credit, linear credit and elastic. The credit approach, constant or linear grants [9] the ONU's requested bandwidth plus an extra amount of bandwidth; while the elastic approach basically limits the maximum cycle time. The rest of the options, fixed, limited and gate dare on prediction-oriented DBAs.

There can be found numerous of issues that an optimum bandwidth prediction scheme can resolve. For example, the prediction scheme should converge fast enough, being also robust to noise. Provided that a prediction model is good, then it should be able to filter out the noisy samples as far as prediction of future bandwidth size is concerned. On the other hand, simple linear prediction scheme is vulnerable to the noisy input. Considering a neural network, in the underlying sequence it cannot adapt quickly to short term structure change. Taking into account the above arguments, a prediction scheme should be able to update the prediction model accordingly, and moreover, detect structural changes in the underlying sequence. Consequently, both the neural network and linear prediction methods do not incorporate properly the correlation structures of the underlying bandwidth size sequence. One of the reasons, why this is happening, is because for each bandwidth size the linear models use separate models. This has as after effect of inability of representation the correlation structures among different types of sizes.

In the distributed approach the participation of both OLT and ONU is required. Bandwidth allocation [10] is calculated by ONU through it is also authorized by OLT. For distributed algorithm the Dynamic Distributed Scheduler for PON (DDSPON) is used.

The DDSPON [6] is a DBA algorithm which is required some extra information mainly the weight vector. This weight vector allows ONUs to compute its transmission window size. Such parameter represents a proportional weight set up according to each ONU's guaranteed bandwidth agreement. The ONU computes the required weight and bandwidth and then reports such value to the OLT in a report message. Moreover, each ONU schedules the size of its transmission window dynamically. DDSPON algorithm [4] is executed in online framework because the scheduling process is executed without the need of waiting the reports from the rest of the ONUs. In addition, by getting the weight vector, each ONU is able to capture the loads of the rest of the ONUs, which is characteristic in offline DBA algorithms.

Hence, the scheduling framework determines the scheduling decisions are made. There are two main frameworks to consider: on-line and off-line scheduling. With online scheduling, the OLT makes scheduling decisions "on-the-fly" based on individual requests and without global knowledge of the network. On the other hand, offline scheduling requires a full knowledge of the network status, thus its scheduling decisions are computed after having received the requests from all of the ONUs [7]. In general, the on line scheme performs better than the offline scheduling, but with less control of channel transmission times

\section{STATUS REPORT DBA (SR-DBA)}

The SR-DBA method [8] is based on queue-status reports sent by ONTs to OLT periodically, as depicted in Fig.3. The SRDBA process consists of the following steps:

- $\quad$ Step 1: Send all SRs from the ONTs to the OLT

- Step 2: Calculation of the bandwidth grant based on DBA algorithm

- $\quad$ Step 3: Sending the upstream bandwidth maps from the OLT to ONTs

- $\quad$ Step 4: Sending of data from the ONUs to the OLT according to the upstream BW maps.

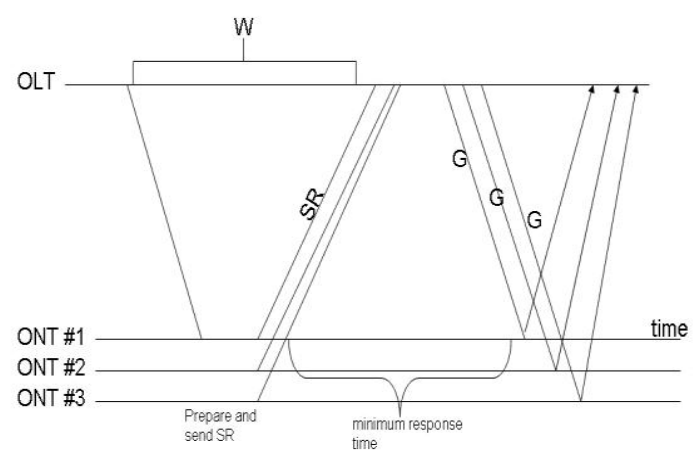

Fig. 3: SR-DBA algorithm

There are many factors which the performance of SR-DBA is affected.

- SR delay time: time taken from the moment a queue is ready for reporting, until, the time taken to be send to the OLT.

- PON round-trip time: affects the SR delay and response time.

- OLT process time: in order to create the next W period from all ONTs the OLT is required to wait for the respective reports.

- Status reports bandwidth: for each status report there is fixed amount of bandwidth allocated. Lower in size $\mathrm{W}$ the higher bandwidth required for these reports.

- W size: even though, the smaller the $\mathrm{W}$ size the better (ideally), on the contrary is has to be large enough the round-trip-time (RTT) of the PON, the status reports bandwidth, and the process time of the OLT. 


\section{BANDWIDTH ALLOCATION (BA- DBA) ALGORITHM}

In this section, the proposed BA-DBA algorithm is presented. The basic concept of BA-DBA algorithm is based on the equal granted amount of bandwidth for each ONT.

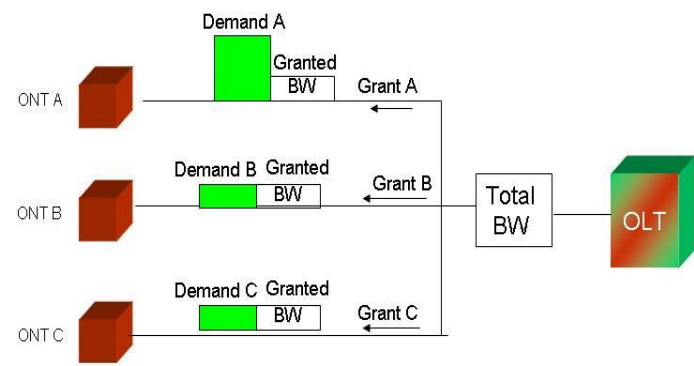

Fig. 4: BA-DBA algorithm

The bandwidth required is distributed among the ONTs in efficient way, where possible. In the following figure the implementation of the BA-DBA algorithm is being presented. The possible scenario and how it works, with the proposed BA-DBA algorithm is shown in fig.5 and fig.6.

In the first transmission, the OLT has total bandwidth of 20Mbps and share in equal amount of $5 \mathrm{Mbps}$ in four ONT units. ONT1 demand 4Mpbs, ONT2 demands 5Mpbs, ONT3 demands $6 \mathrm{Mpbs}$ and ONT4 demands 5 Mbps.

However, ONT3 requests bandwidth more than $5 \mathrm{Mbps}$ and ONT1 less than 5 Mbps. In effect, the extra $1 \mathrm{Mbps}$ from ONT1 will be transferred to ONT3.
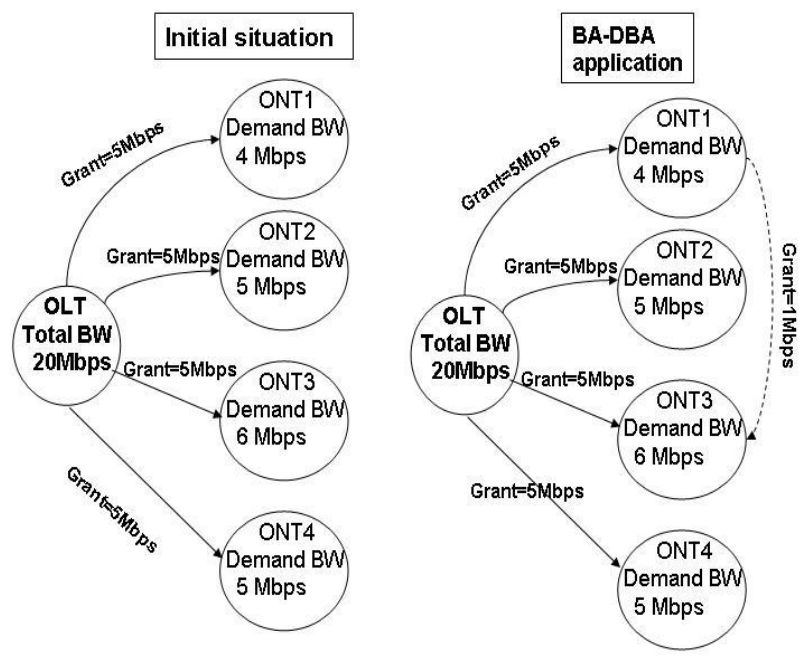

Fig. 5: BA-DBA algorithm (first transmission)

In the second transmission, the OLT has bandwidth $12 \mathrm{Mbps}$, shared in equal amount of $3 \mathrm{Mbps}$ in four ONTs. The required bandwidth of all ONTs is $4 \mathrm{Mbps}$.

ONT3 returns back the extra $1 \mathrm{Mbps}$ and ONT1 transfers the required amount. ONT3 transfers $2 \mathrm{Mbps}$ after transferring the $1 \mathrm{Mbps}$ to ONT1.

The other two, ONT2 and ONT4 are transmit taffic flow in $3 \mathrm{Mbps}$ as assigned.

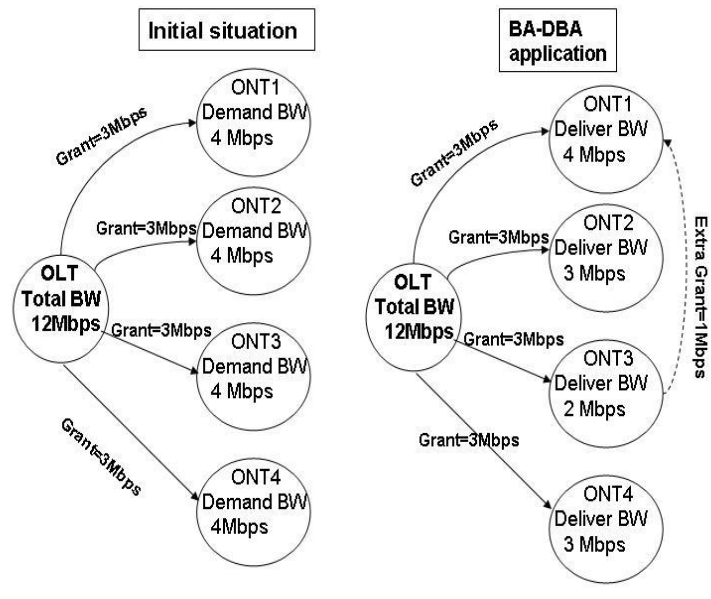

Fig. 6: BA-DBA algorithm (second transmission)

\section{SIMULATION SETUP}

The main idea is to simulate BA-DBA algorithm in a PON network. The simulation program used was NS-2 program [11] run under Linux. The topology which was used / simulated is a tree topology with 16 ONUs, each of them separated from the OLT over the interval from $5 \mathrm{~km}$ to $15 \mathrm{~km}$.

To generate self-similar traffic, Ns-2 provides a traffic source model in which the attributes of the self-similar traffic are specified such as the packet size, the average arrival rate and Hurst parameter. The Hurst parameter was varied from $\mathrm{H}=0,7$ to $\mathrm{H}=0,8$ among different scenarios respectively. The packet size follows a uniform distribution for random size of packets with an under limit of 512 bits and an upper limit of 12144 bits corresponding to the Ethernet frame. To obtain simulations with variations to the ONU offered load, where the total offered load is $1 \mathrm{Gbps}$ and is equally distributed among all active ONUs..

\section{SIMULATION RESULTS}

In this section the simulation results are presented. The scope of the tests is that to verify how to optimize the packet delay, and minimize the effects of the average queue delay using BA-DBA algorithm in comparison with SR-DBA algorithm. Two scenarios of simulations were analyzed in which parameters such as distance and traffic were modified. Table.1 summarizes the simulations parameters used in the simulations experiments.

Table 1: Simulations Parameters

\begin{tabular}{|l|c|c|}
\hline Parameters & Scenario 1 & Scenario 2 \\
\hline Number of ONUs & 16 & 16 \\
\hline OLT line rate & $1 \mathrm{Gbps}$ & $1 \mathrm{Gbps}$ \\
\hline ONU user rate & $100 \mathrm{Mbps}$ & $100 \mathrm{Mbps}$ \\
\hline $\begin{array}{l}\text { Distance OLT-ONU } \\
(\mathrm{km})\end{array}$ & 5 & 15 \\
\hline Hurst traffic parameter & 0.7 & 0.8 \\
\hline Max. cycle time & $1 \mathrm{~ms}$ & $1 \mathrm{~ms}$ \\
\hline $\begin{array}{l}\text { Guard interval } \\
\text { timeslots }\end{array}$ & $.009 \mathrm{~ms}$ & $.009 \mathrm{~ms}$ \\
\hline No of queues per ONU & 1 & 1 \\
\hline
\end{tabular}

The main difference of the two scenarios are the Hurst traffic parameter and the distance between OLT-ONU. The behavior of algorithms are independent from the Hurst parameter and 
only the bandwidth request and distance OLT-ONU influences the simulations. From previous experience, a major percentage of a typical last mile loop is about $2 \mathrm{Km}$, so we consider only the scenario 1 for the simulations.

In the Fig. 7 shows the average queue size in scenario 1 for both algorithms SR-DBA and BA-DBA with various time network loads.

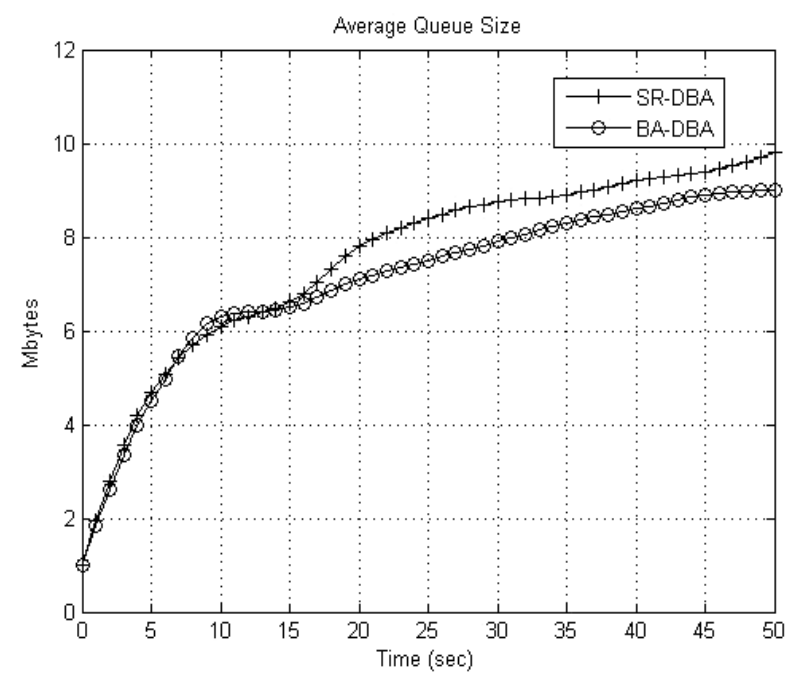

Fig. 7 Average Queue Size

In the figure. 7 , both algorithms experience a similar behavior for low time periods; however, when the time is bigger than $15 \mathrm{sec}$, the SR-DBA algorithm starts to experience an increase in the queue size bigger than our proposed algorithm BADBA.

When the network load reaches its maximum capacity, SRDBA presents an average value of $10 \mathrm{Mbytes}$, whereas BADBA reaches 9 Mbytes. These results have implications in the queue buffer size since, in the case of SR-DBA, it will require to be larger to avoid the packet loss.

In terms of average packet delay, figure. 8 shows the increase of delay as a result of the queue size, i.e. when the network load is increasing, then the queue size starts to increase the delay also increases.

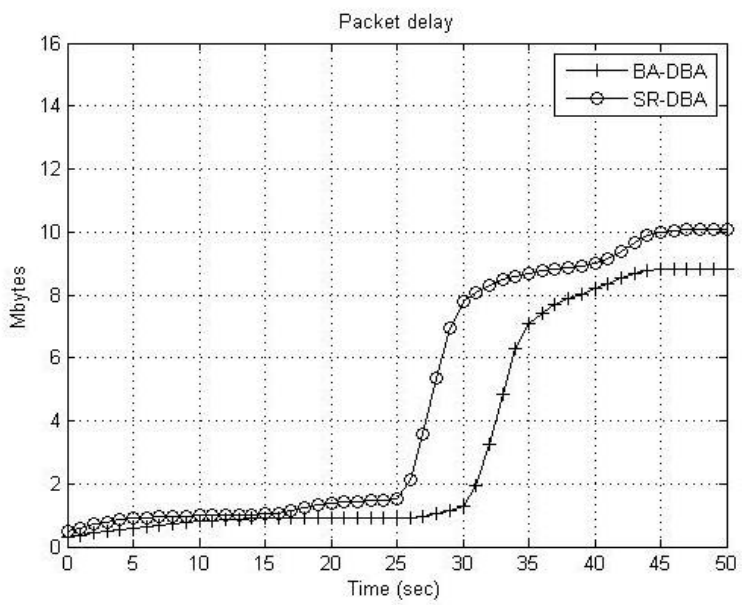

Fig. 8 Packet delay
The proposed BA-DBA algorithm shows lower delays for higher networks loads compared with SR-DBA.

A comparison of the throughput between the two algorithms is presented. Fig. 9 shows the throughput of BA-DBA and SR-DBA.

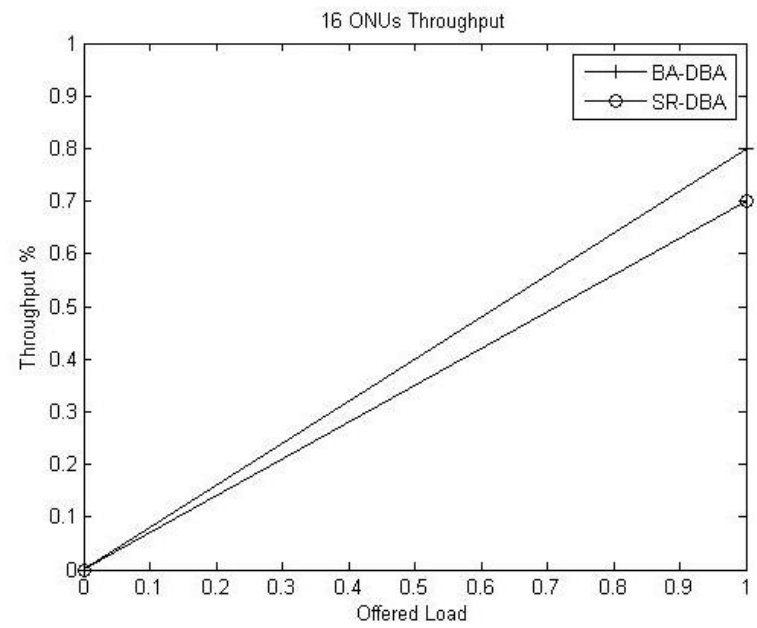

Fig. 9 Throughput BA-DBA vs SR-DBA

As expected both algorithms exploit this property to improve the upstream channel utilization. The proposed algorithm BADBA achieves a throughput of $80 \%$ compared with SR-DBA which achieving $70 \%$.

\section{CONCLUSION}

In this paper, it was described a bandwidth allocation DBA algorithm for PON networks; and enhanced with the comparison with SR-DBA. For both algorithms simulations results have been given on the average queue size and packet delay as a function of the packet size and time. It was shown, that the proposed BA-DBA achieves a better performance compared to SR-DBA. In summary, the use of BA-DBA can be efficiently support the bandwidth allocation of the packets in PON networks.

\section{REFERENCES}

[1] McGarry, M.P.; Reisslein, M. \& Maier, M. (2006). WDM Ethernet passive optical networks. IEEE Communications Magazine, Vol.44, No.2, pp. 15-22.

[2] Hee-Jung Byun; Ji-Myung Nho \& Jong-Tae Lim (2003). Dynamic bandwidth allocation algorithm in Ethernet passive optical networks. Electronics Letters, Vol.39, No.13, pp. 1001-1002

[3] Kramer, G.; Mukherjee, B. \& Pesavento, G. (2002b). Interleaved Polling with Adaptive Cycle Time (IPACT): A Dynamic Bandwidth Distribution Scheme in an Optical Access Network. Photonic Network Communications, Vol.4, No.1, pp. 89-107.

[4] Liu, McGarry, M.P.; Reisslein, M.; Colbourn, C.J.; Maier, M.; Aurzada, F. \& Scheutzow, M. (2008). Just-inTime Scheduling for Multichannel EPONs. Journal of Lightwave Technology, Vol.26, No.10, pp. 1204-1216.

[5] Lannoo, B.; Verslegers, L.; Colle, D.; Pickavet, M.; Demeester, P. \& Gagnaire, M. (2007). Thorough analysis of the IPACT dynamic bandwidth allocation algorithm 
for EPONs. Fourth International Conference on Broadband Communications, Networks and Systems, pp. 486-494.

[6] N. Ansari, a McGarry, M.P.; Maier, M. \& Reisslein, M. (2004). Ethernet PONs: a survey of dynamic bandwidth allocation (DBA) algorithms. IEEE Communications Magazine, Vol.42, No.8, pp. 8-15.

[7] McGarry, M.P.; Reisslein, M.; Colbourn, C.J.; Maier, M.; Aurzada, F. \& Scheutzow, M. (2008). Just-in-Time Scheduling for Multichannel EPONs. Journal of Lightwave Technology, Vol.26, No.10, pp. 1204-1216.

[8] Broadligth White Paper: " The Rigth Method for Dynamic Bandwidth Allocation in Point-to-Multipoint FTTH Networks", Broadligth, 17th July 2003.
[9] Yongqing Zhu; Maode Ma \& Tee Hiang Cheng (2006). IPACT with Grant Estimation for EPON. 10th IEEE Singapore International Conference on Communication systems, 2006. ICCS 2006, pp. 1-5.

[10] Yuanqiu Luo \& Ansari, N. (2005). Bandwidth allocation for multiservice access on EPONs. IEEE Communications Magazine, Vol.43, No.2, pp. 16-21.

[11] Ns-2, “ The network simulator ns-2", http://www.isi.edu/nsma/ns (current 6 June 2011) 